\title{
A CERTAIN MEAN-VALUE PROBLEM IN STATISTICS*
}

\author{
BY A. T. CRAIG
}

1. Introduction. It is the purpose of this paper to investigate, by means of the characteristic function, the arithmetic mean value, or mathematical expectation, of the sum of the squares of $n$ normally and independently distributed variables when those variables are subject to $m<n$ linear restrictions. For example, if $x_{1}, x_{2}, \cdots, x_{n}$ are $n$ independent values of a variable $x$ which is normally distributed with mean zero and variance $\sigma^{2}$, then the expected value of $\sum_{1}^{n} x_{j}{ }^{2}$ is $n \sigma^{2}$. However, the expected value of $\sum_{1}^{n}\left(x_{1}-\bar{x}\right)^{2}$, where $n \bar{x}=\sum_{1}^{n} x_{j}$, is $(n-1) \sigma^{2}$. It is fairly obvious that the latter example could be stated: if the $x$ 's are subject to the linear restriction $\sum_{1}^{n} x_{j}=0$, the expected value of $\sum_{1}^{n} x_{j}{ }^{2}$ is $(n-1) \sigma^{2}$. The numbers $n$ and $n-1$, which are equal respectively to the ranks of the matrices of the two quadratic forms, are frequently called the number of degrees of freedom of those quadratic forms.

Let $x$ be subject to the normal law of error

$$
f(x)=\frac{1}{\sigma(2 \pi)^{1 / 2}} e^{-x^{2} / 2 \sigma^{2}}
$$

and let $x_{1}, x_{2}, \cdots, x_{n}$, be $n$ independent values of $x$. Write

$$
v=\sum_{1}^{n} x_{j}^{2}, \quad u_{1}=\sum_{1}^{n} a_{1 j} x_{j}, \cdots, \quad u_{m}=\sum_{1}^{n} a_{m j} x_{j},
$$

in which the $a$ 's are real numbers. We wish to find the mathematical expectation of $v$ when $u_{1}, u_{2}, \cdots, u_{m}$ are assigned values which make the system consistent. It is well known that the variables $u_{1}, u_{2}, \cdots, u_{m}$ are normally correlated with variances and covariances given by $\sigma^{2} \sum_{r} a_{j r} a_{k r}$.

2. The Characteristic Function. The characteristic function of the joint distribution of $v, u_{1}, \cdots, u_{m}$ is

* Presented to the Society, April 11, 1936. 


$$
\phi\left(t_{1}, t_{2}, \cdots, t_{m}, t_{m+1}\right)=\left(\frac{1}{2 \pi \sigma^{2}}\right)^{n / 2} \int \cdots \int e^{\theta} d x_{n} \cdots d x_{1}
$$

where

$$
\theta=i t_{1} \sum_{1}^{n} a_{1 j} x_{j}+\cdots+i t_{m} \sum_{1}^{n} a_{m j} x_{j}+\left(i t_{m+1}-\frac{1}{2 \sigma^{2}}\right) \sum_{1}^{n} x_{j}{ }^{2},
$$

and $i=\sqrt{ }(-1)$. Throughout this paper we shall understand that the limits of integration are $-\infty$ and $\infty$ unless otherwise specified. If we write

$$
\begin{aligned}
& b_{11}=\sum a_{1 j}^{2}, b_{22}=\sum a_{2 j}^{2}, \cdots, b_{m m}=\sum a_{m j}^{2}, \\
& b_{12}=b_{21}=\sum a_{1 j} a_{2 j}, \cdots, b_{m-1, m}=b_{m, m-1}=\sum a_{m-1, j} a_{m j},
\end{aligned}
$$

and

$$
Q=\sum_{j, k} b_{j k} t_{j} t_{k}
$$

then

$$
\phi\left(t_{1}, \cdots, t_{m+1}\right)=\frac{e^{-\sigma^{2} Q / 2\left(1-2 i \sigma^{2} t_{m+1}\right)}}{\left[1-2 i \sigma^{2} t_{m+1}\right]^{n / 2}} .
$$

From this latter result, it is fairly obvious that the problem has no solution unless $Q$ is a positive definite quadratic form of rank $m$. Upon writing $t_{m+1}=0$, we find the characteristic function of the joint distribution of the $m$ linear forms to be

$$
\phi\left(t_{1}, \cdots, t_{m}, 0\right)=e^{-\sigma^{2} Q / 2} .
$$

Moreover, if $\psi=\psi\left(u_{1}, \cdots, u_{m}\right)$ is the simultaneous distribution function of these linear forms, then

$$
\psi=\left(\frac{1}{2 \pi}\right)^{m} \int \cdots \int e^{-L-\sigma^{2} Q / 2} d t_{m} \cdots d t_{1},
$$

where

$$
L=i t_{1} u_{1}+\cdots+i t_{m} u_{m} .
$$

Since $Q$ is positive definite of rank $m$, the Cayley-Hamilton equation of the matrix $B=\left\|b_{j k}\right\|$ of $Q$ has $m$ real positive roots, say $\lambda_{1}, \lambda_{2}, \cdots, \lambda_{m}$. Moreover, there exists a real orthogonal matrix $C=\left\|c_{j k}\right\|$ such that 


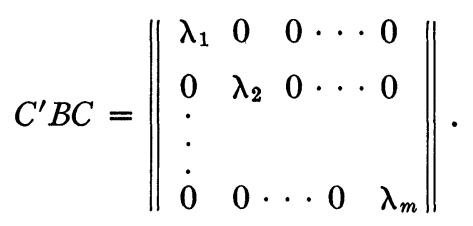

If then, in the latter integral, we introduce new variables $z_{1}, \cdots, z_{m}$ by subjecting the $t$ 's to a linear homogeneous transformation with matrix $C$, we get

$$
\begin{aligned}
\psi & =\left(\frac{1}{2 \pi}\right)^{m} \int \cdots \int e^{-i S_{1} z_{1}-\cdots-i S_{m} z_{m}-\left(\sigma^{2} / 2\right) \Sigma \lambda_{j} z_{j}^{2}} d z_{m} \cdots d z_{1} \\
& =\frac{1}{\left(\lambda_{1} \cdots \lambda_{m}\right)^{1 / 2}\left(2 \pi \sigma^{2}\right)^{m / 2}} e^{-S_{1}{ }^{2} / 2 \lambda_{1} \sigma^{2}-\cdots-S_{m}{ }^{2} / 2 \lambda_{m} \sigma^{2}}
\end{aligned}
$$

where $S_{p}=\sum c_{p j} u_{j},(p=1,2, \cdots, m)$.

3. The Mathematical Expectation of v. Let $F=F\left(u_{1}, \cdots, u_{m}, v\right)$ be the simultaneous distribution function of $v$ and the $m$ linear forms. Also, let $\bar{v}$ be the expected value of $v$ for $u_{1}, \cdots, u_{m}$ assigned. Thus

$$
\bar{v}=\int \frac{v F}{\psi} d v
$$

in which the limits of integration on $v$ are here and elsewhere taken to cover all admissible values of that variable when $u_{1}, \cdots, u_{m}$ are regarded as assigned. Now

$$
\phi\left(t_{1}, \cdots, t_{m}, t_{m+1}\right)=\int \cdots \int e^{L+i v t_{m+1} F} d v d u_{m} \cdots d u_{1},
$$

and

$$
\begin{aligned}
\left.\frac{\partial \phi}{\partial t_{m+1}}\right|_{t_{m+1}=0} & =i \int \cdots \int v e^{L} F d v d u_{m} \cdots d u_{1} \\
& =i \int \cdots \int v e^{L} \frac{F}{\psi} \psi d v d u_{m} \cdots d u_{1} \\
& =i \int \cdots \int \bar{v} e^{L} \psi d u_{m} \cdots d u_{1} .
\end{aligned}
$$

Thus 


$$
i \bar{v} \psi=\left.\left(\frac{1}{2 \pi}\right)^{m} \int \cdots \int e^{-L} \frac{\partial \phi}{\partial t_{m+1}}\right|_{t_{m+1}=0} d t_{m} \cdots d t_{1} .
$$

But

$$
\left.\frac{\partial \phi}{\partial t_{m+1}}\right|_{t_{m+1}=0}=i\left(n \sigma^{2}-Q \sigma^{4}\right) e^{-\sigma^{2} Q / 2} .
$$

Accordingly,

$$
\begin{aligned}
\bar{v} \psi=\left(\frac{1}{2 \pi}\right)^{m} \int \cdots \int\left(n \sigma^{2}-Q \sigma^{4}\right) e^{-L-\sigma^{2} Q / 2} d t_{m} \cdots d t_{1} \\
=n \sigma^{2} \psi-\sigma^{2} \psi\left[\left(1-\frac{S_{1}^{2}}{\lambda_{1} \sigma^{2}}\right)+\left(1-\frac{S_{2}^{2}}{\lambda_{2} \sigma^{2}}\right)+\cdots\right. \\
\left.\quad+\left(1-\frac{S_{m}^{2}}{\lambda_{m} \sigma^{2}}\right)\right]
\end{aligned}
$$

and

$$
\bar{v}=\sigma^{2}\left[n-m+\frac{1}{\sigma^{2}}\left(\frac{S_{1}^{2}}{\lambda_{1}}+\cdots+\frac{S_{m}^{2}}{\lambda_{m}}\right)\right] .
$$

We now see that if each linear form is set equal to zero, the expected value of $v$ is $\bar{v}=(n-m) \sigma^{2}$. Thus, when $u_{1}=u_{2}=\cdots=u_{m}$ $=0$, we may say that we lose one degree of freedom for each linear restriction in estimating $\sigma^{2}$ from $v$.

4. Independent Linear Restrictions. Of particular interest is the case in which the variables $u_{j}$ are not correlated. A necessary and sufficient condition for the independence of the variables $u_{j}$ is that

$$
\begin{aligned}
& \phi\left(t_{1}, 0, \cdots, 0,0\right) \cdot \phi\left(0, t_{2}, 0, \cdots, 0\right) \cdots \phi\left(0, \cdots, 0, t_{m}, 0\right) \\
&=\phi\left(t_{1}, \cdots, t_{m}, 0\right) ;
\end{aligned}
$$

that is, when $b_{j k} \neq 0, j=k$, and $b_{j k}=0, j \neq k$. Under these conditions, $\psi$ becomes

$$
\psi=\left(\frac{1}{2 \pi \sigma^{2}}\right)^{m / 2} \frac{1}{\left(b_{11} \cdots b_{m m}\right)^{1 / 2}} e^{-u_{1}^{2} / 2 \sigma^{2} b_{11}-\cdots-u_{m}^{2} / 2 \sigma^{2} b_{m m}}
$$

and 


$$
\bar{v}=\sigma^{2}\left[n-m+\frac{1}{\sigma^{2}}\left(\frac{u_{1}^{2}}{b_{11}}+\cdots+\frac{u_{m}^{2}}{b_{m m}}\right)\right] .
$$

Again we observe that the expected value of $v$ is $(n-m) \sigma^{2}$ when each of the $m$ linear forms is equated to zero. However, if $s$ of the $m$ linear forms are equated to their respective standard derivations while the remaining $m-s$ are equated to zero, then $\vec{v}=(n-m+s) \sigma^{2}$. Finally we see that the expected value of $v$, for a fixed set of $u$ 's, is not in general an integral multiple of $\sigma^{2}$.

The University of Iowa

\section{ON THE PRESERVATION OF ANGLES AT A BOUNDARY POINT IN CONFORMAL MAPPING $\dagger$}

\section{BY S. E. WARSCHAWSKI}

The object of this note is to prove the following theorem.

THEOREM. Let $R$ be a simply connected "schlicht" region in the $w$-plane whose boundary contains the point $w=0$. Let $w=0$ be "accessible" along the Jordan curve L. Suppose that there is a circle $|w|<\rho$ such that the part of the boundary of $R$ which is inside this circle lies within the angles

$$
\left|\arg w-h_{+}\right| \leqq k_{+}, \quad\left|\arg w-h_{-}\right| \leqq k_{-}, \quad\left(h_{-} \leqq h_{+}\right) .
$$

Suppose, furthermore, that $L$ connects $w=0$ with a boundary point outside $|w|=\rho$ such that $L$ divides $R$ into two sub-regions. Let all boundary points of one sub-region which are in $|w|<\rho$, and not on $L$, be in one of the angles (1), and those of the other sub-region which are in $|w|<\rho$, and not on $L$, be in the other.

Let $w=w(z)$ map $|z-1|<1$ conformally on $R$ in such a manner that its inverse function approaches 0 as $w \rightarrow 0$ along $L$. Let

$$
\begin{aligned}
& H(\alpha)=\frac{1}{\pi}\left[\left(\frac{\pi}{2}+\alpha\right) h_{+}+\left(\frac{\pi}{2}-\alpha\right) h_{-}\right], \\
& K(\alpha)=\frac{1}{\pi}\left[\left(\frac{\pi}{2}+\alpha\right) k_{+}+\left(\frac{\pi}{2}-\alpha\right) k_{-}\right] .
\end{aligned}
$$

$\dagger$ Presented to Society, October 26, 1935. 